\title{
Multiple local feature representations and their fusion based on an SVR model for iris recognition using optimized Gabor filters
}

\author{
Fei He ${ }^{1,2}$, Yuanning Liu ${ }^{1,2}$, Xiaodong Zhu ${ }^{1,2^{*}}$, Chun Huang ${ }^{1,2}$, Ye Han ${ }^{1,2}$ and Hongxing Dong ${ }^{1,2}$
}

\begin{abstract}
Gabor descriptors have been widely used in iris texture representations. However, fixed basic Gabor functions cannot match the changing nature of diverse iris datasets. Furthermore, a single form of iris feature cannot overcome difficulties in iris recognition, such as illumination variations, environmental conditions, and device variations. This paper provides multiple local feature representations and their fusion scheme based on a support vector regression (SVR) model for iris recognition using optimized Gabor filters. In our iris system, a particle swarm optimization (PSO)and a Boolean particle swarm optimization (BPSO)-based algorithm is proposed to provide suitable Gabor filters for each involved test dataset without predefinition or manual modulation. Several comparative experiments on JLUBR-IRIS, CASIA-I, and CASIA-V4-Interval iris datasets are conducted, and the results show that our work can generate improved local Gabor features by using optimized Gabor filters for each dataset. In addition, our SVR fusion strategy may make full use of their discriminative ability to improve accuracy and reliability. Other comparative experiments show that our approach may outperform other popular iris systems.
\end{abstract}

Keywords: Iris recognition; Gabor filters; Local Gabor features; Boolean particle swarm optimization; Support vector regression

\section{Introduction}

The first iris recognition system was proposed by Daugman in 1993 and is still the state-of-the-art technique used today [1,2]. Wildes' iris biometric method is another important approach [3,4]. Subsequently, a large number of algorithms have been developed to develop an iris practical system with less control [5-7]. However, the commercial iris recognition system still has problems, such as intra-class variations (e.g., iris texture affected by ageing), inter-class similarities (leads to false acceptance), and noise in data (e.g., illumination effect to iris image pixels) [8].

Feature extraction is a crucial stage for addressing these problems [9]. Two options exist to improve iris recognition performance. One option is to find effective and fast iris representation for various acquisition

\footnotetext{
*Correspondence: zhuxd@jlu.edu.cn

${ }^{1}$ College of Computer Science and Technology, Jilin University, Qianjin Street, Changchun 130012, China

2 Key Laboratory of Symbolic Computation and Knowledge Engineering of Ministry of Education, Jilin University, Qianjin Street, Changchun 130012, China
}

conditions. Daugman presented iris texture using Gabor phase-based code that is invariant under the non-affine elastic distortion [2]. Huang et al. proposed a new rotation invariant iris feature based on the non-separable wavelet and the Markov random model [10]. These methods analyzed iris texture properties in the frequency domain based on Fourier and wavelet transforms. However, these methods have the disadvantage of fixed transform kernels and cannot pertinently match the changing nature of different iris datasets. Some researchers select an optimal features subset based on filtered coefficients to enhance their distinctive information [11], but what these researchers adopted for filtering is still a predefined log-Gabor wavelet. Chang et al. discovered an unconventional approach that applied an improved empirical mode decomposition (EMD) method. The EMD method is a multi-resolution decomposition technique without any predetermined filter or wavelet function to the iris pattern extraction [12]. Moreover, several approaches exist that directly scan geometric descriptors at the iris images. Mehrotra et al. selected iris local features using the scale

\section{Springer}

(c) 2014 He et al.; licensee Springer. This is an Open Access article distributed under the terms of the Creative Commons

Attribution License (http://creativecommons.org/licenses/by/4.0), which permits unrestricted use, distribution, and reproduction in any medium, provided the original work is properly credited. 
invariant feature transform (SIFT) [13]. However, several key problems, such as illumination variations, environmental conditions, and device variations, cannot be fully addressed using a single form of iris feature.

The second option is to extract multiple iris features and then combine them to compensate for the weakness of a single feature in particular situations. Raja Sekar et al. presented a fusion method of statistical and cooccurrence features that were extracted from the curvelet and ridgelet transformed images. The Manhattan distance and the multiclass classifier with a logistic function were used to generate the final classification result [14]. Tan et al. utilized ordinal measures, color analysis, texture representation, and semantic information as iris features as well as the weighted sum rule to generate the fused score for classification [15]. Gong et al. selected three wavelength bands to represent an iris and then integrated them using agglomerative clustering based on a two-dimensional principal component analysis [16]. The fusion of multiple features is regarded as a positive step towards the development of extremely ambitious types of iris recognition [17]. However, the fusion of multiple features should overcome the challenge of the heterogeneous manifestation of various features.

In this paper, we focus on both options described above to handle the challenges of illumination variation, environmental conditions, and device variations in iris recognition. To find more effective iris features, we use optimized Gabor filters instead of fixed Gabor kernels to characterize iris texture information. Because Gabor transformation can achieve the best trade-off in spatial and frequency resolution [18], several iris systems characterize iris features using Gabor coefficients after Daugman's introduction [19-21]. Most of these algorithms require predefined Gabor basis functions, but when we tried to determine the Gabor parameters in the Gabor function, different options emerged from the existing literatures [22-25] (Table 1), which suggests that no unified pattern of Gabor filters exists for various texture representations. We applied empirical Gabor filters on our selfdeveloped JLUBR-IRIS dataset to the CASIA-V4-Interval dataset to extract the same Gabor features. The obtained distance distribution histograms (DDHs) on these two

Table 1 The Gabor filter selection from different literatures on the CASIA-I dataset

\begin{tabular}{lcccc}
\hline $\begin{array}{l}\text { First } \\
\text { author }\end{array}$ & $\begin{array}{c}\text { Covered frequency } \\
\text { band }\end{array}$ & $\begin{array}{c}\text { Scaling } \\
\text { factor }\end{array}$ & $\begin{array}{c}\text { Scale } \\
\text { number }\end{array}$ & $\begin{array}{c}\text { Orientation } \\
\text { number }\end{array}$ \\
\hline $\mathrm{Li}[22]$ & 32 & 2 & 4 & 4 \\
$\mathrm{Ma}[23]$ & 64 & 2 & 6 & 4 \\
$\mathrm{Nabti}[24]$ & $\mathrm{N} / \mathrm{A}$ & $\mathrm{N} / \mathrm{A}$ & 4 & 6 \\
$\mathrm{Li}[25]$ & $\mathrm{N} / \mathrm{A}$ & $\mathrm{N} / \mathrm{A}$ & $\mathrm{N} / \mathrm{A}$ & 4 \\
\hline
\end{tabular}

datasets are shown in Figures 1 and 2. The enormous difference in the performance of the datasets demonstrates that no universally applicable Gabor filters exist for all the datasets. Motivated by this, we preferred to extract further informative iris texture features by adopting suitable Gabor filters. However, explicit solution was seldom found in the existing literature for obtaining the proper Gabor parameters for its basic function. Several researchers executed the Gabor filter selection based on several predefined candidate filters [26,27]. The abovedescribed approaches require that their candidates cover and target the most informative band (MIB) of involved iris samples. These approaches achieved excellent results on certain iris datasets, but cannot be adapted to arbitrary iris datasets. Chou et al. reduced the free Gabor parameters by preserving only the horizontal frequency components and achieved the optimal multi-scale Gabor filters by using the Gene Algorithm [28]. This method has a faster optimization process, while the problems of local optimum and texture loss surfaced. Tsai et al. tuned the parameters of a Gabor filter by using the particle swarm optimization (PSO) algorithm to generate the optimal Gabor filters one by one [29].

The algorithm benefited from the immense global searching ability of PSO and can obtain an optimal single Gabor filter. However, the whole Gabor filter set may not be effective. Possible overlaps may exist among the Gabor bands and may include redundant information for the Gabor features. To address this problem, we propose a Gabor filter optimization algorithm based on PSO and its binary version Boolean PSO (BPSO). In our work, the lower dimensional parameters of the Gabor filter set with an orthogonal kernel constraint condition are analyzed. For the real values and integer values in Gabor set parameters, PSO and BPSO rules are utilized to obtain the optimal Gabor bands for each involved dataset. Our method can adaptively determine the amount of Gabor filters, bandwidth, and covered frequency bandwidth to generate the orthogonal Gabor filters in place of manual

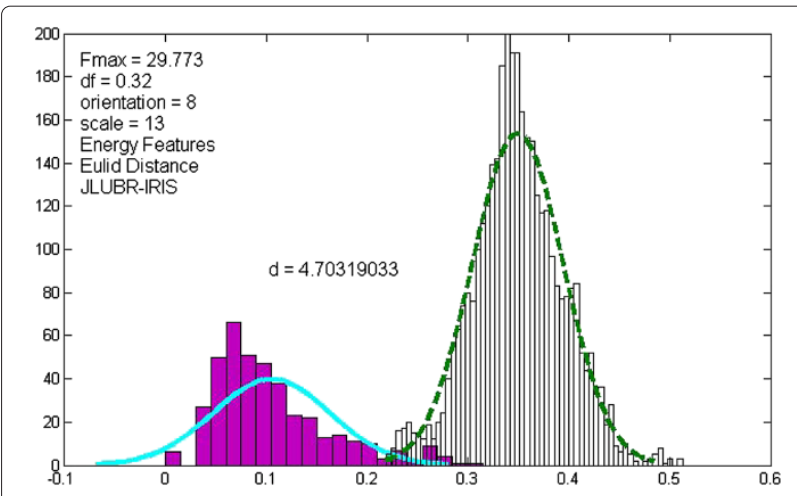

Figure 1 DDH for the JLUBR-IRIS dataset. 


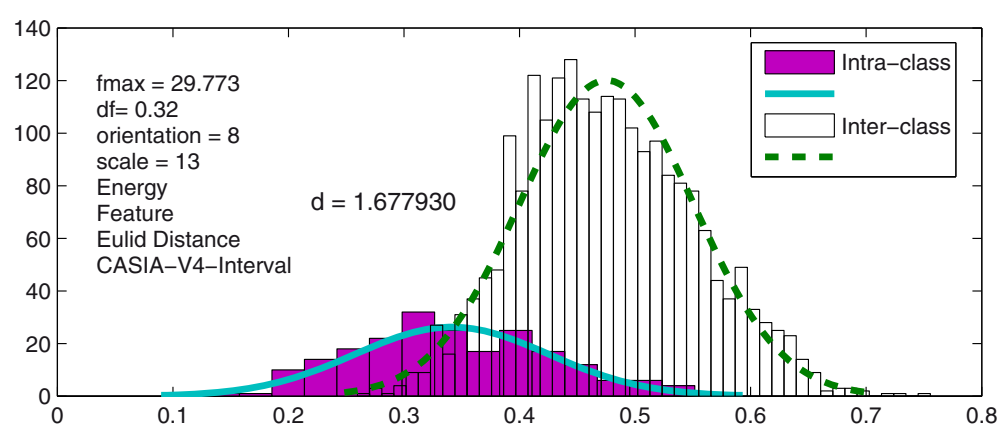

Figure 2 DDH for the CASIA-V4-Interval dataset.

modulation. The optimized Gabor filters may cater to the diverse frequency coverage of various iris datasets and are related to the capture device, acquisition condition, and individual physiology. Moreover, local features can offer a closer analysis of the uniqueness of the iris texture but are generally included in the irregular distribution of the iris image blocks such as crypts, freckles, coronas, stripes, and furrows [30]. Therefore, we divided the Gabor response magnitude and phase to generate local feature vectors, where iris texture can be further preserved. Compared with the traditional localized mode of iris image division, our localized method occurred after convolution and may avoid the blocking effect in the process of image division.

To fuse multiple iris features, we extract two different types of Gabor features to describe the iris texture from the energy spectrum and frequency domain and then combine them using a new non-linear fusion strategy. The Gabor response magnitude is the model of orientation for the selective neuron in the primary visual cortex [31] and is related to the local energy spectrum, while the Gabor phase can capture the information from the wavelet's zero crossing [2]. An advantage of our multiple feature extraction is lower computational complexity because both features can be calculated by only one Gabor transformation. Furthermore, in the process of Gabor transformation, DCfree Gabor kernel is adopted because of its invariance property to the ambient illumination [32], while Gabor phase features of irises are assigned regardless of how low the image contrast, illumination, and camera gain [33]. Thereby, our proposed system involved extensive Gabor energy features that may perform more robustness and reliability when illumination variation exists. For the combination of the two kinds of Gabor features, we prefer a more flexible score level fusion as opposed to a feature level fusion, which needs to address the heterogeneity of various features, and a decision level fusion, which involves less information [34]. The match score is a real value measure of the similarity between the input and template biometric feature vectors. In score level fusion, all real value scores from multiple features will be combined into a real value to arrive at a final recognition decision. A robust and effective score fusion method based on

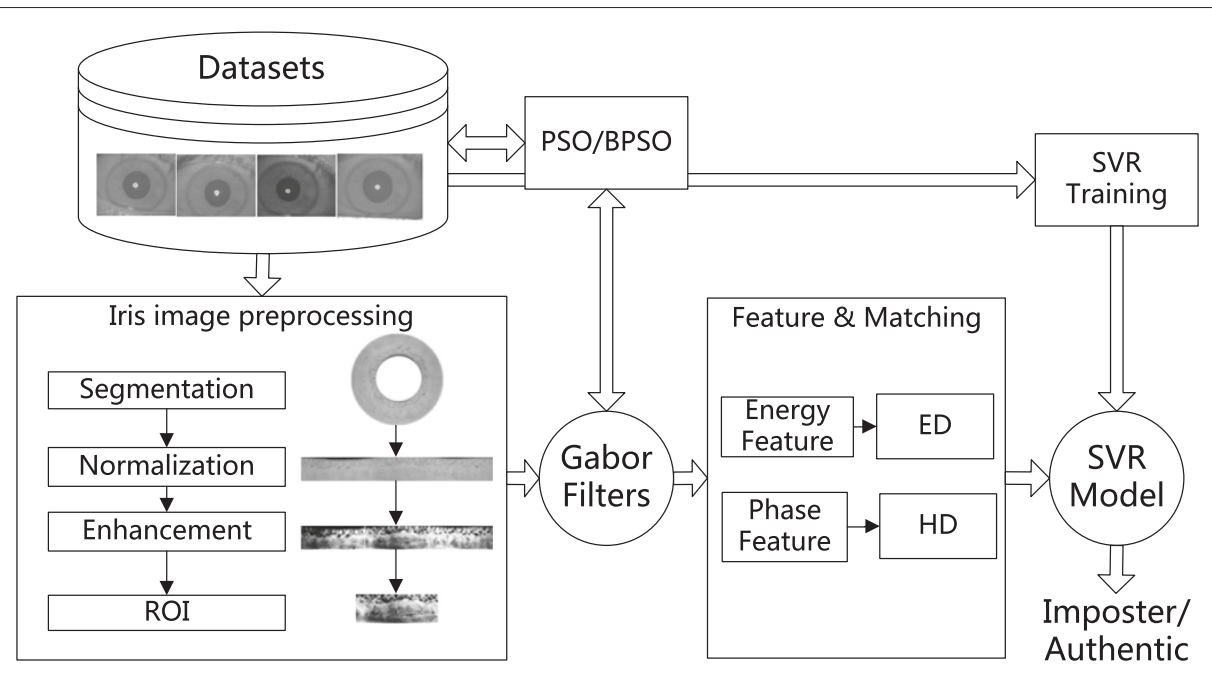

Figure 3 The architecture of the proposed iris recognition system. 


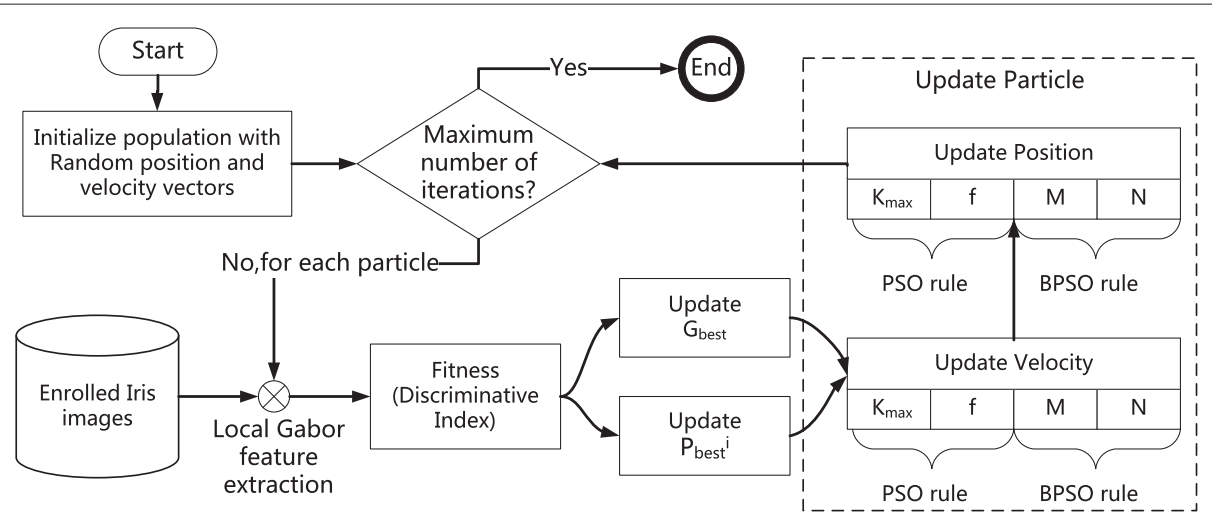

Figure 4 The flowchart of the Gabor filter optimization by PSO and BPSO.

support vector regression (SVR) is proposed in this paper. This method may fuse matching scores from different features using a non-linear and high-dimensional regression function, which will better fit the non-correlations among matching scores from multiple features.

The architecture of the proposed iris recognition system is shown in Figure 3. It is generally known that a captured iris image contains not only the meaningful texture part but also some un-useful parts (e.g., eyelid and pupil) [22]. So, separating the meaningful region, called region of interest (ROI), from the captured iris images should be carried out. Otherwise, the extra noises will be taken in following stages and affect the effectiveness of iris feature extraction. In our system, we separate the iris region from the iris captured images by the Canny operator and the Hough transformation [35] and then employ the ROI extraction of $\mathrm{Yu}$ et al. to provide more precise iris templates [22]. This method uses the longest-chordbased method to determine the location of the pupil and then uses a circular template to search the outer boundary of the ROI. During the feature extraction stage, the local Gabor energy and phase representations are produced by the same Gabor filters with parameters that have been optimized by the proposed PSO and BPSO. Next, all matching scores from both Gabor features are sent into a trained SVR model and are mapped to a single scalar score to make the final decision.

The remainder of this paper is organized as follows: Section 'Gabor filters' introduces the generation process of two types of local Gabor features and their matching scores. Section 'Gabor filter optimization by PSO and BPSO' illustrates the Gabor filter optimization by PSO and BPSO. Section 'Score fusion scheme based on SVR' describes multiple Gabor feature fusion schemes based on SVR. Section 'Experimental results' presents the experimental setup and results. Finally, Section 'Conclusion' concludes the paper.

\section{Gabor filters}

\section{Gabor function}

Various expressions of the Gabor basic function exist in the spatial and frequency domains. Compared with the frequency expression, the spatial expression can be directly represented in a discrete form for the convolution

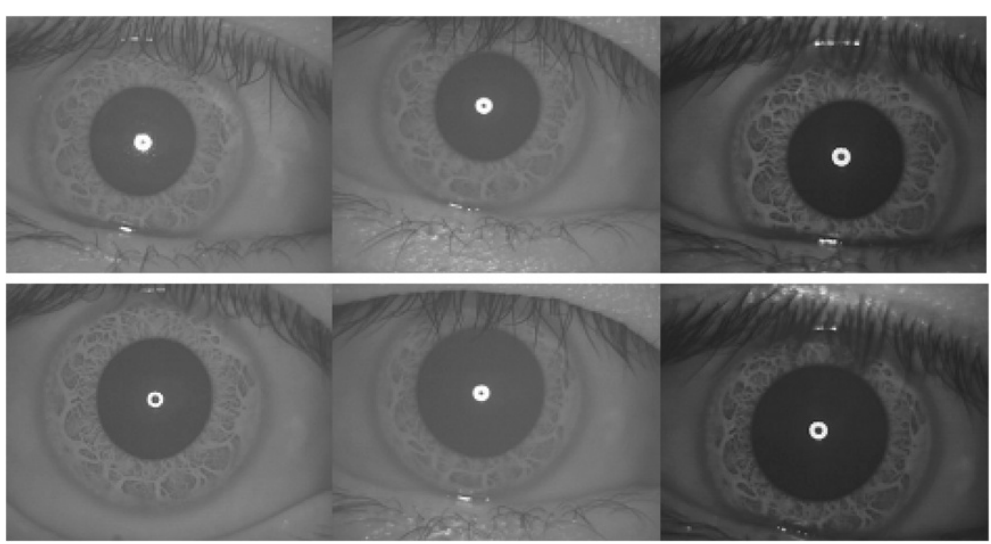

Figure 5 Samples from different acquisition times and six illumination levels. 
Table 2 Dataset description

\begin{tabular}{lccccc}
\hline Number & Dataset & Class & $\begin{array}{c}\text { Samples } \\
\text { per class }\end{array}$ & $\begin{array}{c}\text { Enrollment } \\
\text { per class }\end{array}$ & $\begin{array}{c}\text { Test } \\
\text { per class }\end{array}$ \\
\hline 1 & CASIA-I & 50 & 7 & 4 & 3 \\
2 & CASIA-V4-Interval & 50 & 7 to 10 & 5 & 2 to 5 \\
3 & JLUBR-IRIS & 50 & 45 & 25 & 20 \\
\hline
\end{tabular}

of iris images. The Gaussian envelope should fall completely into discrete bins so that the filtered coefficient values are negligible outside a limited length discrete filter [36]. In the spatial domain, a general function of the two-dimensional Gabor kernel is defined as follows [22]:

$$
G_{\mu, v}(z)=\frac{\left\|k_{\mu, \nu}\right\|^{2}}{\sigma^{2}} e^{-\frac{\left\|k_{\mu, \nu}\right\|^{2}\|z\|^{2}}{2 \sigma^{2}}}\left[e^{i k_{\mu, \nu} z}-e^{-\frac{\sigma^{2}}{2}}\right]
$$

From Equation 1, we can see that the Gabor function is a product of a Gaussian envelope and a complex plane wave, where $\mu$ and $v$ determine the objective orientation and scale, respectively. The center of the receptive field is $z=(x, y)$. The norm operator is $\|\bullet\|$. The standard derivation of the Gaussian envelope is $\sigma$, which determines the ratio of the Gaussian width to the wavelength. We adopt the DC-free Gabor kernel that offers an invariance property to the ambient illumination change in the iris image acquisition [37]. The wave vector is defined as follows:

$$
\begin{aligned}
k_{\mu, \nu} & =k_{\nu} e^{i \phi_{\mu}} \\
e^{i k_{\mu, v^{2}}} & =\cos \left(k_{\mu, \nu} z\right)+i \sin \left(k_{\mu, \nu} z\right),
\end{aligned}
$$

where $k_{v}$ and $\phi_{\mu}$ are the frequency and orientation of targeted texture.

\section{Multi-channel Gabor filters}

A set of multi-channel Gabor filters should be used to provide the basis for distinguishing irises [38]. Due to the non-uniform frequency distribution of the iris texture and to obtain isotropy in the orientations, we choose transfer functions that have size variations and lattice discretization [39]. The selection of frequency $k_{v}$ in Equation 4 can be computed as follows:

$$
k_{\nu}=K_{\max } / f^{v} \quad v=0,1, \cdots, M-1,
$$

where $K_{\max }$ is the maximum frequency that defines the covered frequency band, $f$ is the frequency scaling factor, and $M$ defines the number of all extracted scales. An important wavelet property that provides the orthogonal basis to Gabor functions is inherited by Equation 4 [38]. The standard derivation $\sigma$ is $\sigma=K_{\max } / 2\left(2^{M}-1\right)$. The selection of targeted angles is $\phi_{\mu}$ calculated as follows:

$$
\phi_{\mu}=\mu \pi / N \quad \mu=1,2, \cdots, N
$$

In that, $N$ is the number of targeted orientations. Therefore, a quadruple of Gabor parameters $\left\{K_{\max }, f, M, N\right\}$ determines a set of Gabor filters.

\section{Two types of localized Gabor features}

In this paper, we extract two different types of local Gabor features based on the Gabor responses and their division. To generate local Gabor features, using Gabor response division instead of image division can eliminate the blocking effect in the process of convolution. The blocking effect of image division will lead some staircase noises into Gabor transformation [40]. If several iris characteristics exist in a localized block, they will be degraded such that the block boundary looks like the edge. Further, the accuracy and reliability of the Gabor features will be badly hurt.

One type of localized Gabor feature in our work is generated by dividing each Gabor response magnitude into $r \times c$ size blocks. The statistical means of all blocks constitute a local energy Gabor feature because the Gabor response magnitude is related to the local energy spec-
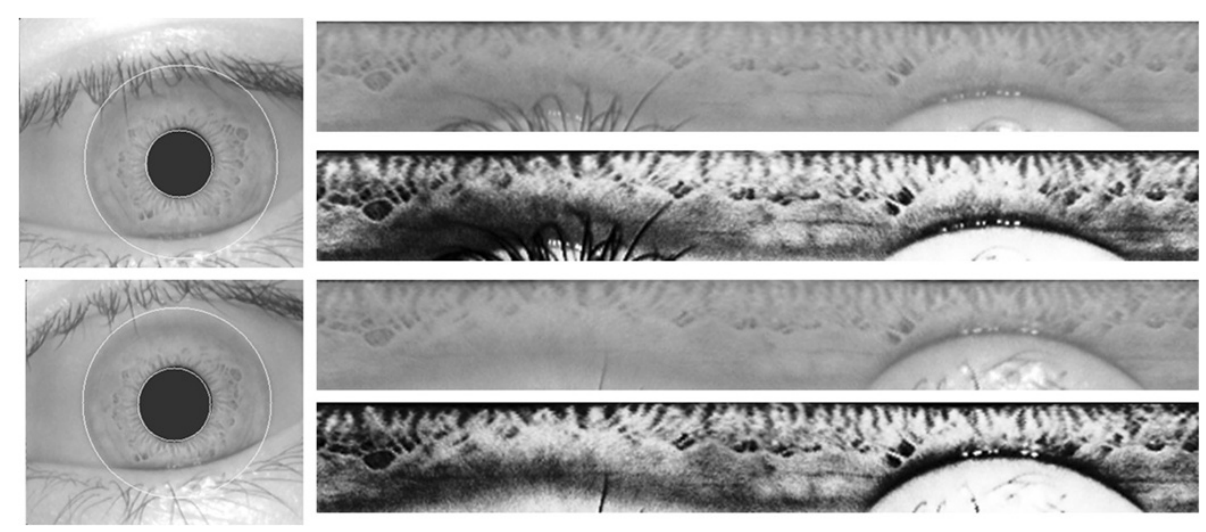

Figure 6 An example of false non-match due to eyelid and eyelash occlusion on the CASIA-I dataset. 

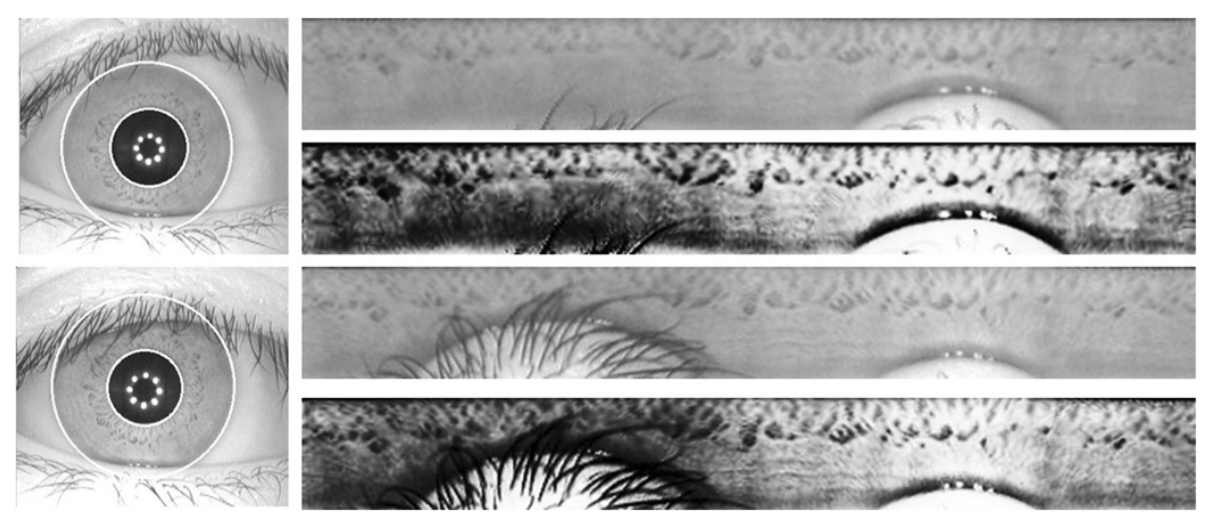

Figure 7 An example of false non-match due to eyelid and eyelash occlusion on the CASIA-V4-Interval dataset.

trum. The matching score may be calculated using the Euclidean distance (ED). To eliminate the effects of dimension, the $L_{2}$ norm of each iris feature may be designated in the ED computation.

The second type of localized Gabor feature, called the local Gabor phase feature, is generated by dividing each Gabor response phase into $r \times c$ size blocks. Next, each block is encoded in accordance with the Daugman rule [1]. Hamming distance (HD) is used to compute across a population of unrelated phase codes bit by bit.

\section{Gabor filter optimization by PSO and BPSO The concepts of PSO and BPSO}

As a swarm intelligence method, PSO was developed by Kennedy and Eberhart [41] and has been a remarkable technique for optimization problems in a real value field due to its strong global search capability [42]. In PSO, a population of particles $P_{i}=\left(p_{i, 1}, p_{i, 2}, \cdots, p_{i, n}\right)$ is initialized for an optimal solution by updating their values with its own velocity $V_{i}=\left(v_{i, 1}, v_{1,2}, \cdots, v_{i, n}\right)$. In recursions, all particles and their velocities are replaced by the best previous position of the current particle $P_{\text {best }}{ }^{i}$ and the best previous position of all particles $G_{\text {best }}$ as follows:

$$
\begin{aligned}
v_{i, j}(t+1)= & w \times v_{i, j}(t)+c_{1} \times r_{1} \times\left(P_{i, j}^{\text {best }}-p_{i, j}(t)\right) \\
& +c_{2} \times r_{2} \times\left(G_{\text {best }}-p_{i, j}(t)\right) j=1,2, \cdots, n
\end{aligned}
$$

$p_{i, j}(t+1)=p_{i, j}(t)+v_{i, j}(t+1)$,

where $p_{i, j}$ is the $i$ th and $j$ th dimensional particle. The inertial weight is $w$ and is generated in the range $[0,1][43]$. The velocity $v_{i, j}$ is restricted to the range $\left[-V_{\max }, V_{\max }\right]$.

A fitness function is defined to evaluate the position of the particles. After a limited number of recursions, the particle that satisfies the global best fitness is chosen as the optimal result.

Despite the successful application of PSO in a real number field, the updated strategy of velocity and position cannot be directly applied to our Gabor filter optimization due to the discrete search field of parameters $M$ and $N$. Kennedy and Eberhart provided the concept and principle of PSO in a discrete domain, named BPSO [44]. In $\mathrm{BPSO}$, the definition of velocity is developed into a prob-
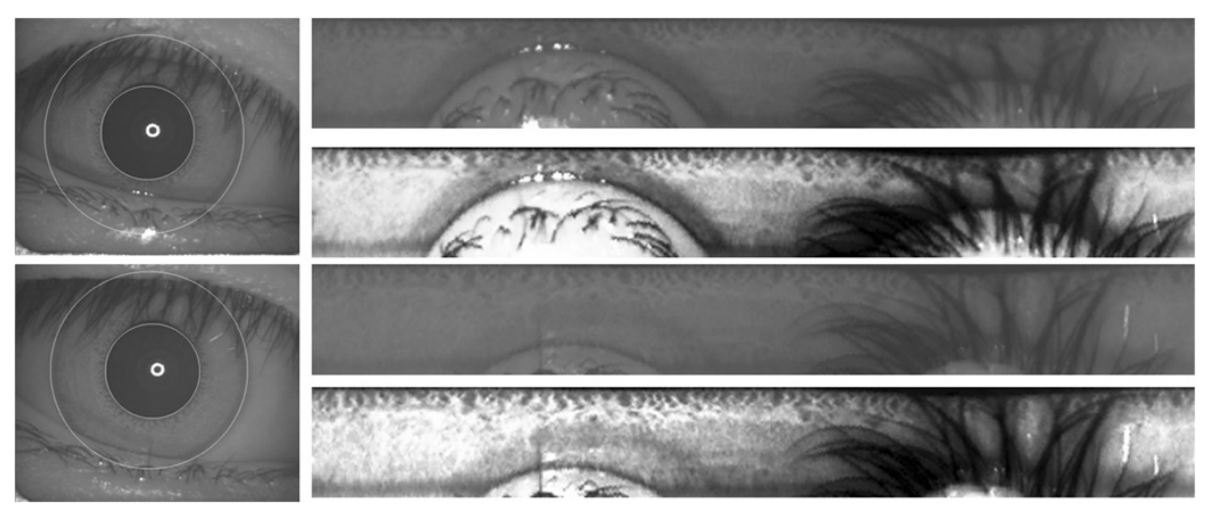

Figure 8 An example of false non-match due to eyelid and eyelash occlusion on the JLUBR-IRIS dataset. 


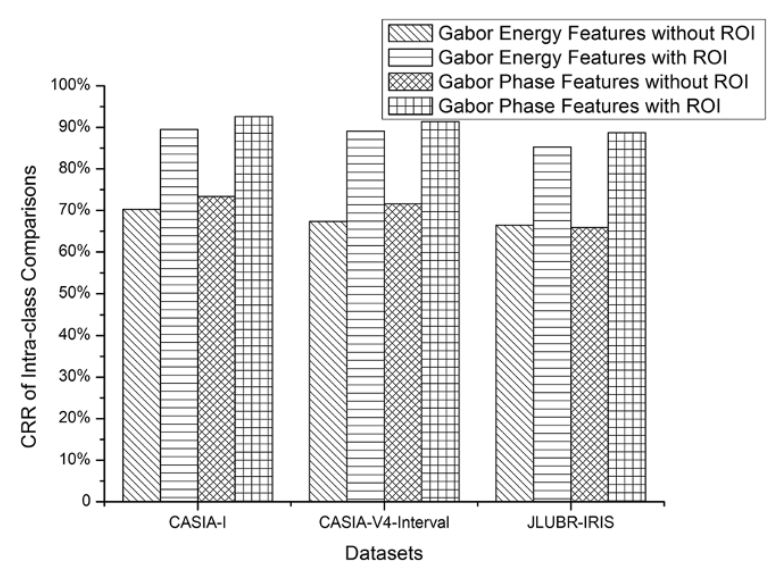

Figure 9 The comparisons of the test images with ROI and without ROI extraction.

ability in which a certain bit position will receive 1 value [45]. Equations 8 and 9 are rewritten as follows:

$$
\begin{aligned}
v_{i, j}(t+1)= & c_{1} \otimes\left(P_{i, j}^{\text {best }} \oplus p_{i, j}(t)\right) \\
& +c_{2} \otimes\left(G_{\text {best }} \oplus p_{i, j}(t)\right) j=1,2, \ldots, n
\end{aligned}
$$

and

$$
p_{i, j}(t+1)=p_{i, j}(t) \oplus v_{i, j}(t+1),
$$

where $\oplus$ means the 'xor' operator, and $\otimes$ and + are the 'and' and 'or' operators, respectively. $\mathrm{c} 1$ and $\mathrm{c} 2$ control the probability that every bit of $\left(P_{i, j}^{\text {best }} \oplus p_{i, j}(t)\right)$ and $\left(G_{\text {best }} \oplus\right.$ $\left.p_{i, j}(t)\right)$ will take 1 value. The constraint of maximum velocity still exists in BPSO but limits the number of 1-value bits in velocity.

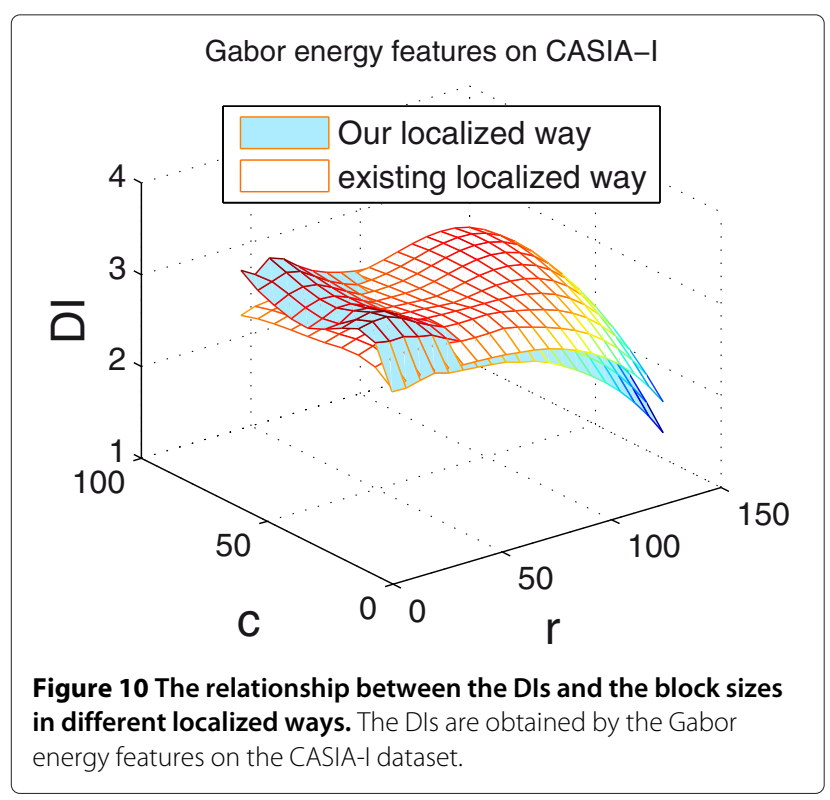

\section{Gabor filter optimization by PSO and BPSO}

In the implementation of Gabor filter optimization, four Gabor parameters formed four-dimensional position of each particle. At the initial phase, we set the population of particles as 10 and the maximum number of iterations as 100. After initialized random values to the position and velocity of all particles, all enrollments of iris sample are involved in filtering with the Gabor filters represented in each particle to extract local Gabor energy and phase features. We thus can quantitatively assess the discriminative ability of the Gabor energy and phase features as fitness. The indicator used for evaluation in our work is the discriminative indexes (DIs) as follows:

$$
d=\frac{\left|\mu_{\text {inter }}-\mu_{\text {intra }}\right|}{\sqrt{\frac{\sigma_{\text {inter }}^{2}+\sigma_{\text {intra }}^{2}}{2}}},
$$

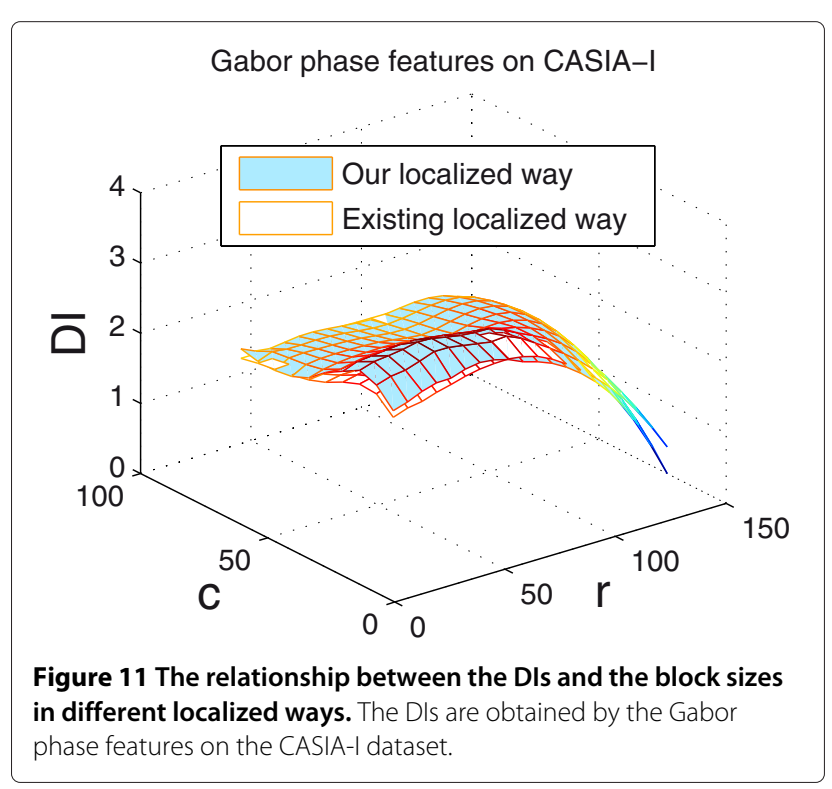




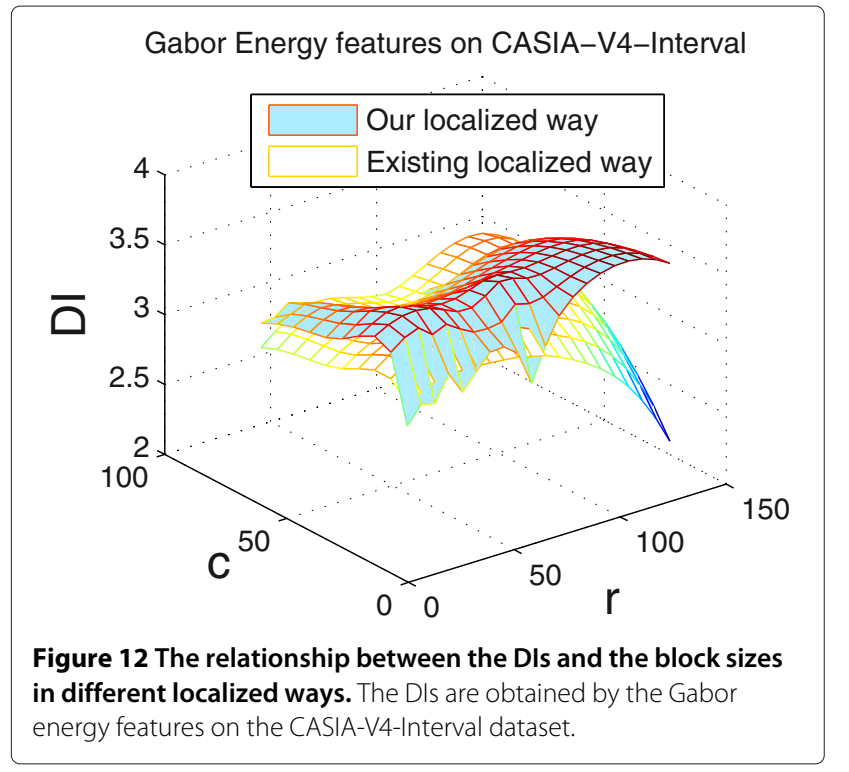

where $\mu$ and $\sigma^{2}$ denote the mean and variance of interclass and intra-class distance, respectively. The fitness of an optimal objective therefore can be defined as follows:

$$
\text { fitness }=\alpha \cdot d_{\text {energy }}+(1-\alpha) \cdot d_{\text {phase }},
$$

where $d_{\text {energys }}$ and $d_{\text {phase }}$ are the DI values, respectively, produced by the Gabor energy and phase features according to the Gabor filters represented in each particle. The larger the fitness points, the better the position in the problem space. In this paper, optimized Gabor filters should achieve a trade-off of performance between the energy feature and the phase feature, so that the scale factor $\alpha$ is set 0.5 . In each recursion, according to their

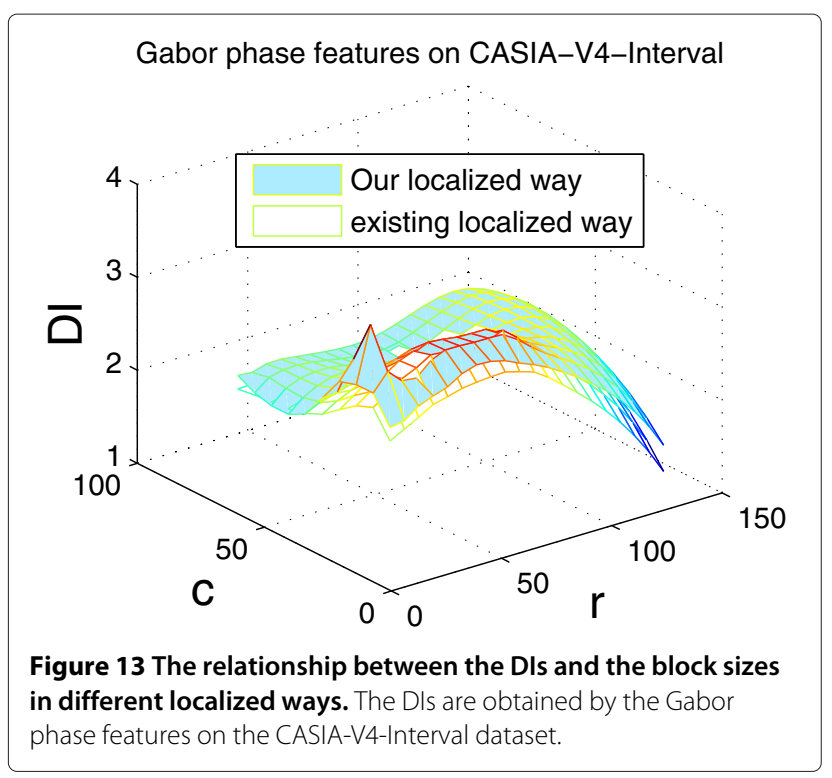

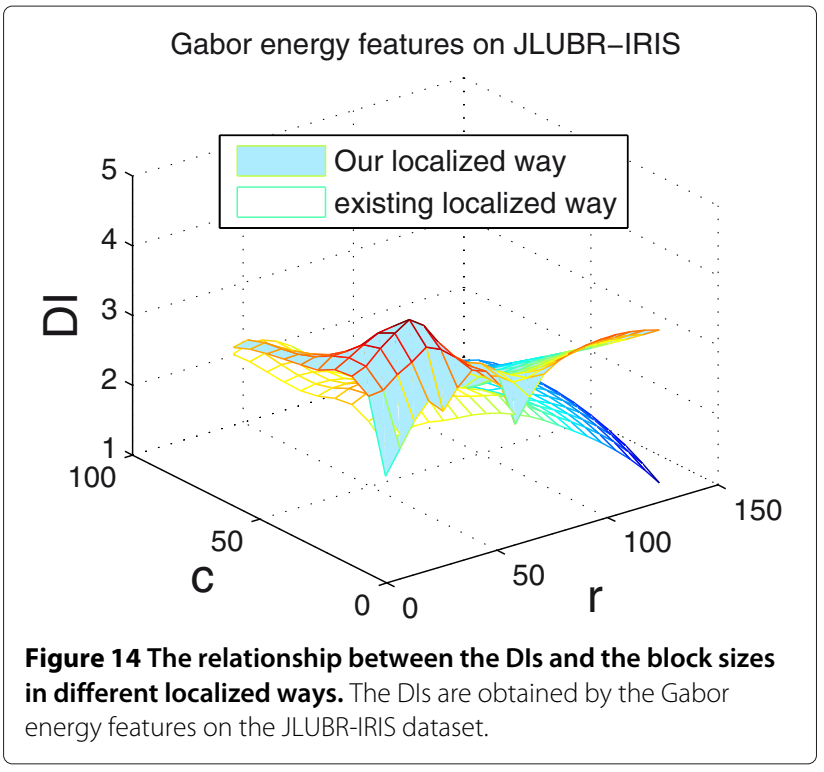

fitness values, we can determine the best previous position of each particle $P_{\text {best }^{i}}$ and the best previous position of all particles $G_{\text {best }}$ to update the velocity and the position of all particles. During the execution of iterations, two real-number parameters $K_{\max }$ and $f$ will follow the PSO rules, while the discrete parameters $M$ and $N$ follow the BPSO rules. At the end of the iterations, all we have to do for determining the best particle is just to observe their fitness values of all particles and figure out the particle with the largest fitness value. The best fitness suggests that its corresponding Gabor filters best fit the MIB of the involved iris dataset, as they generate local Gabor energy and phase features with greatest distinctive ability. The

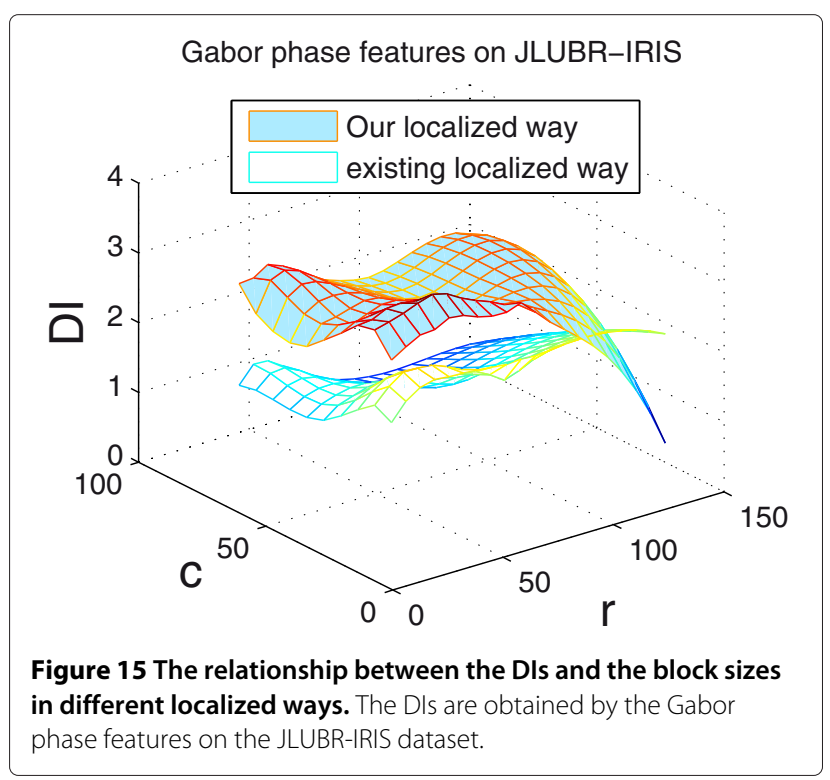




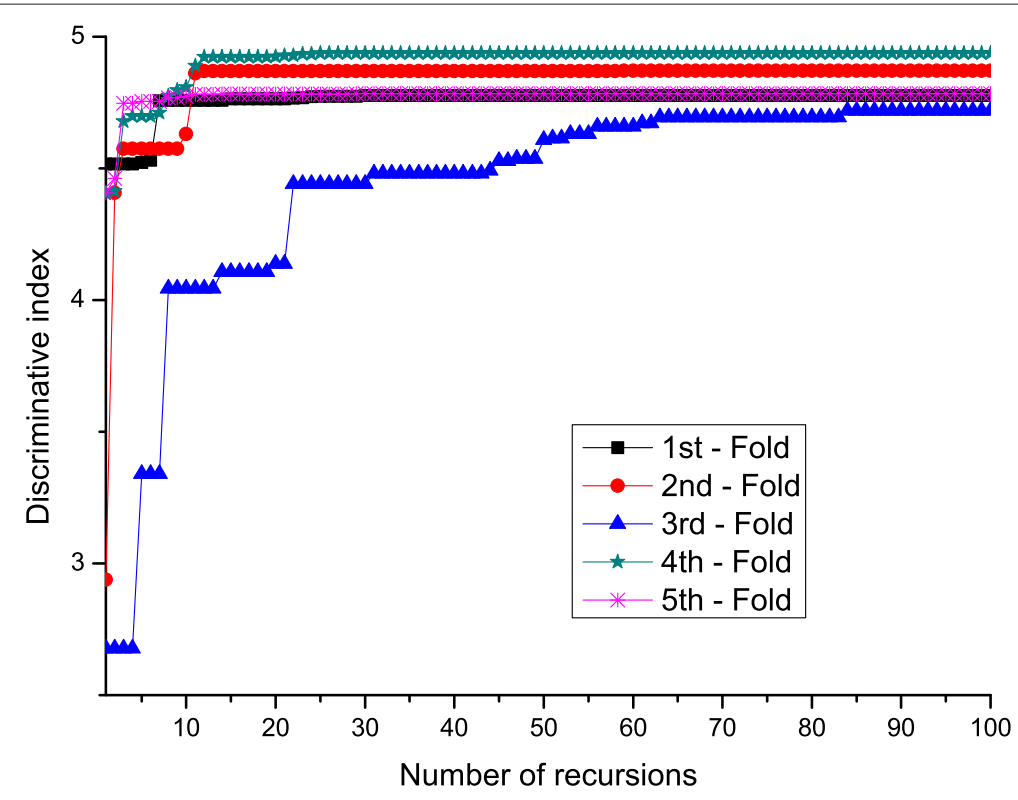

Figure 16 The fitness values of PSO and BPSO on the CASIA-I dataset.

flowchart of the Gabor filter optimization algorithm is shown in Figure 4.

\section{Score fusion scheme based on SVR}

After we achieve the most appropriate Gabor filters to extract most informative energy and phase features, the combination of their discriminative ability should be considered. In score fusion level, the linear weighted strategy is frequently used in some literatures [46,47]. In our work, due to the uncorrelated matching scores from the Gabor energy and phase features, a non-linear fusion rule based on SVR is adopted. The idea of SVR is based on the computation of a regression function in a high-dimensional feature space where the input data are mapped via a nonlinear function [48]. The regression function $f(x)$ in SVR can be denoted as follows:

$$
f(x)=\sum_{i=1}^{k}\left(\beta_{i}^{*}-\beta_{i}\right) K\left(x, x_{i}\right)+b \quad i=1,2, \ldots, k
$$

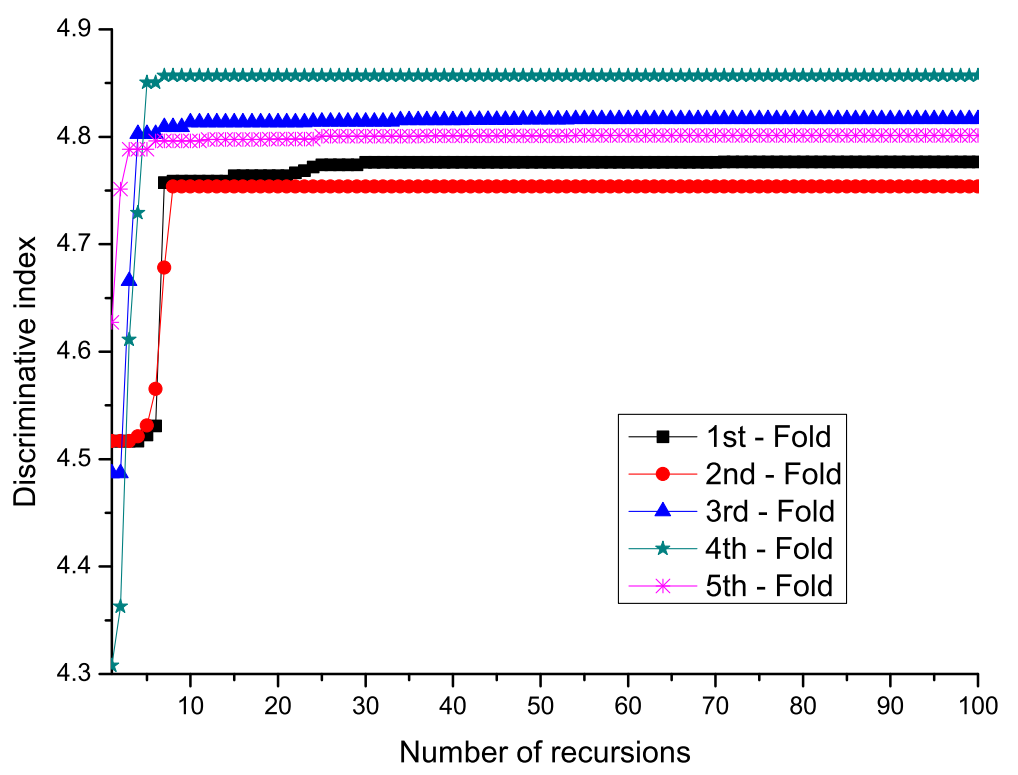

Figure 17 The fitness values of PSO and BPSO on the CASIA-V4-Interval dataset. 


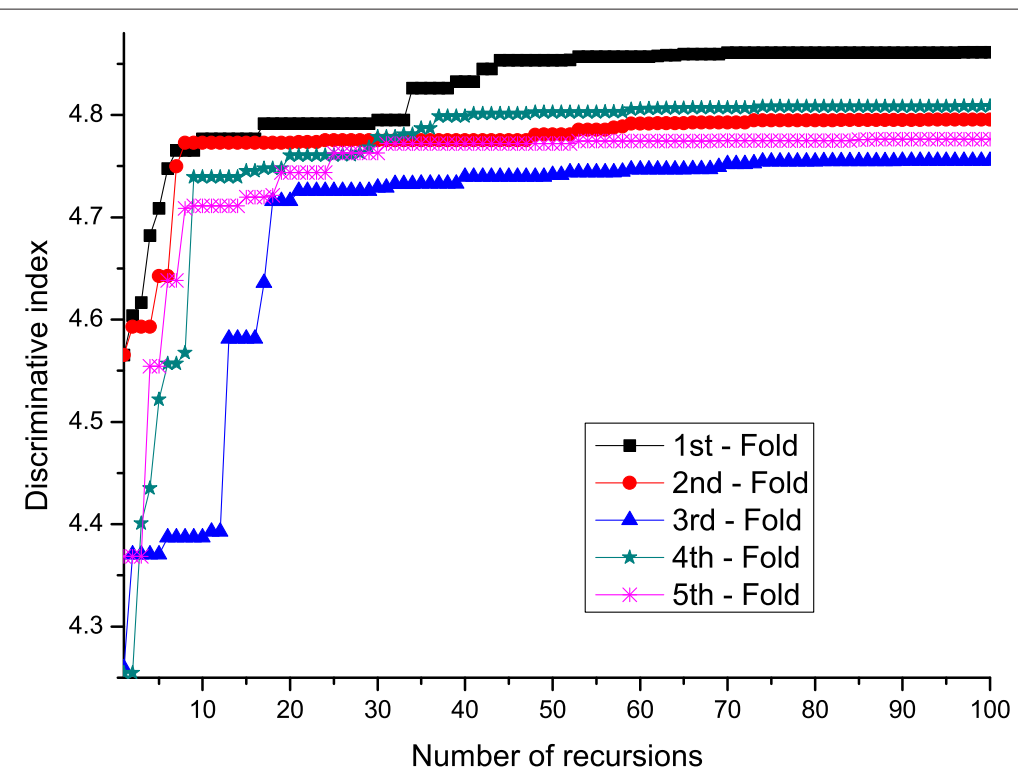

Figure 18 The fitness values of PSO and BPSO on the JLUBR-IRIS dataset.

where $k$ is the number of training data. The Lagrangian multipliers $\beta_{i}^{*}, \beta_{i}$ are found by solving a quadratic programming problem [49], and $b$ is the bias. A kernel function $K(u, v)$ performs the non-linear mapping. Any symmetric function that satisfies Mercer's condition can be chosen as $K(u, v)$. The usual kernels include dot, polynomial, radial basis function (RBF), and neuron kernels [50].

We take advantage of the SVR to fit a function $f(x)$, which may map multiple matching scores to a fused score to make the final decision of arbitrary one-to-one identity. The ED and HD of the Gabor energy and phase features in a comparison are formed as an input vector, both of which have been normalized to the $[0,1]$ real-value range. This intrinsic characteristic of matching scores just might naturally avoid the question of heterogeneous input of fusion. To train a SVR model, all input data with labels from arbitrary one-to-one comparisons of enrolled irises are used to train an SVR model. The authentic comparisons will be labeled 0 as the observed value, while the imposter comparisons will be labeled 1 . In the forecast mode of trained SVR, an input score vector may be mapped to a real value as its fused score. This value can be considered to integrate multiple local Gabor features to measure similarity between two irises. A lower value (close to the authentic label) obtained by output of the SVR demonstrates that the test iris and the involved enrolled iris are in the same pattern class. In light of this principle, only a reasonable threshold should be chosen to complete the classification decision.

\section{Experimental results \\ Datasets}

For the purpose of sufficiently investigating iris recognition performance under changing illuminations, acquisition deflection, ageing, and other circumstances, we established a new JLUBR-IRIS dataset using our self-developed online iris image capture system [51]. Informed, written consent was obtained from all participants for iris collection. The images in the JLUBR-IRIS dataset were gathered under various illumination levels from indoors. A class of samples from different acquisition times and different illumination levels is shown in Figure 5.

In this paper, three datasets, including the JLUBR-IRIS, CASIA-I, and CASIA-V4-Interval datasets, are used to examine the effectiveness of the proposed algorithm. Due to the weaker contrast of texture in the Asian iris as opposed to the European iris, all experimental subjects from the three chosen datasets are Chinese people to

Table 3 Optimized fitness and optimized Gabor filters on three datasets

\begin{tabular}{|c|c|c|c|c|c|c|c|c|}
\hline \multirow[t]{2}{*}{ Dataset } & \multicolumn{2}{|c|}{ DI/predefined Gabor } & \multicolumn{2}{|c|}{ DI/optimized Gabor } & \multicolumn{4}{|c|}{ Gabor parameters } \\
\hline & Energy & Phase & Energy & Phase & $K_{\max }$ & $f$ & $M$ & $N$ \\
\hline CASIA-I & 3.213 & 3.102 & 3.893 & 3.906 & 38.199 & 0.800 & 6 & 31 \\
\hline CASIA-V4-Interval & 3.007 & 3.164 & 3.704 & 3.618 & 19.504 & 0.569 & 6 & 27 \\
\hline JLUBR-IRIS & 3.030 & 2.570 & 4.173 & 3.785 & 10.125 & 0.333 & 12 & 7 \\
\hline
\end{tabular}


Table 4 The DI values from the fused scores via NU-SVR with four kernels on three datasets

\begin{tabular}{lcccc}
\hline Dataset & \multicolumn{4}{c}{ Kernels } \\
\cline { 2 - 5 } & Linear & Poly & RBF & Sigmoid \\
\hline CASIA-I & 4.178 & 2.710 & 4.422 & 0.797 \\
CASIA-V4-Interval & 3.488 & 2.124 & 3.947 & 1.248 \\
JLUBR-IRIS & 2.642 & 4.183 & 3.563 & 0.486 \\
\hline
\end{tabular}

control comparability $[52,53]$. Table 2 shows the experimental settings for the three datasets.

Every iris image in Table 2 is manually selected from accurate iris region segmentation by the Canny operator and the Hough transformation [35] to prevent interference caused by iris misalignment.

\section{Results}

First, we would like to report the experiments that explain the necessity of ROI extraction in our system. As stated in the above description, several noises including eyelashes may occlude the effective regions of the iris for feature extraction and cause false non-matching in intra-class comparisons [54]. Moreover, the various sizes of eyelids in normalized iris images also may damage the similarity between two irises from a pattern class. The typical examples from the two main conditions on three datasets are illustrated in Figures 6,7,8. We designate the three iris datasets within ROI and without ROI extraction for the experiments using local Gabor energy and phase features and observe the effect of precise ROI segmentation on iris recognition performance. In order to achieve unbiased results, the comparative experiments within ROI and without ROI extraction are based on the same fixed Gabor filters $\left(K_{\max }=64, f=2, M=6, N=4\right)$ introduced in the literature [23] and the same localized block size. Their obtained correct recognition rate (CRR) of intraclass comparisons can be found in Figure 9. As the experimental results show, $19.28 \%, 21.73 \%$, and $19.88 \%$ false non-matches of intra-class comparisons can be prevented by the ROI extraction when using Gabor energy features on three iris datasets, respectively. Meanwhile 19.31\%,
$19.87 \%$, and $22.84 \%$ false non-matches of intra-class comparisons can be reduced when using Gabor phase features on three iris datasets, respectively. It means that all kinds of useful iris feature extraction should be performed only when the ROI region may be functioned well and the redundant eyelids and eyelashes can be excluded from the stage. The ROI extraction is thus an indispensable part in our system. The greatest improvement of CRR emerges on JLUBR-IRIS among three iris datasets, which implies that the iris images from this dataset contain more challenging disturbances including eyelids and eyelashes.

Next, we initialized the comparison between the different localized feature extraction methods for the three adopted iris datasets. We also use the fixed Gabor filters $\left(K_{\max }=64, f=2, M=6, N=4\right)$ to extract the Gabor features. In this test, we divide the ROI images to generate features that represent the existing localized method [55]. Various grid search-based block sizes are used to analyze two localized ways. Figures 10,11,12,13,14,15 shows the relationships between DIs and block sizes in two localized means. From Figures 10,11,12,13,14,15, the smallest block size is not the most suitable for localized features, and if the block size is focused excessively on the minute texture, the local features will not enhance the iris texture information but will include redundant noises. Therefore, the block size of localization has to be adjusted for different batch samples. Furthermore, for Gabor energy and phase features, our proposed localized way can obtain more powerful local features and can reveal that our localized approach may conserve more texture in the process of image division and convolution. In all of the following experiments, the localized block size that obtains the best discriminative ability will be adopted.

Put the performance on three datasets all together and we can find that the local Gabor features, extracted by using the chosen Gabor filters, perform better on CASIA-I and CASIA-V4-Interval than JLUBR-IRIS obviously. In the next experiment, we therefore attempted to implement the Gabor filter optimization based on PSO and BPSO with the enrollments of three datasets. To obtain unbiased Gabor filters, we conducted fivefold experiments to achieve the best fitness among them as

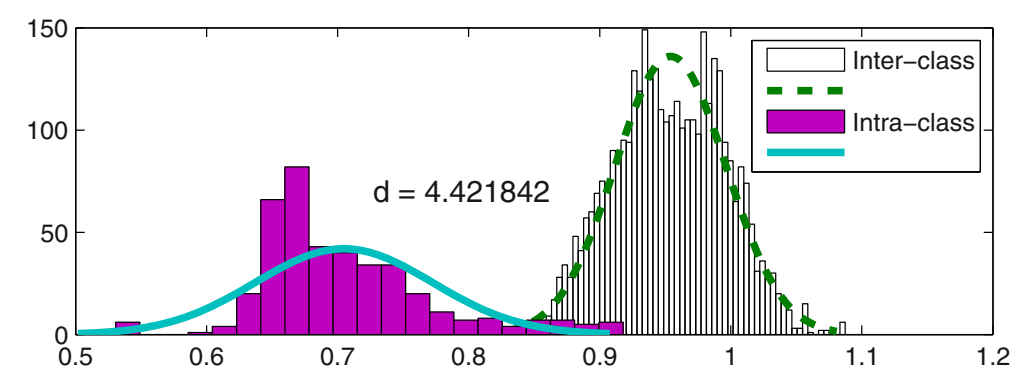

Figure 19 The DDH of fused scores on the CASIA-I dataset. 


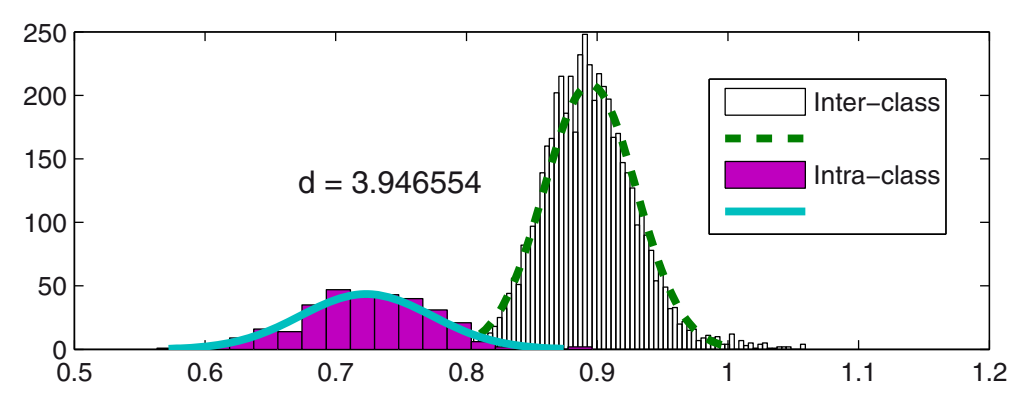

Figure 20 The DDH of fused scores on the CASIA-V4-Interval dataset.

the optimal results. The optimization details of PSO and BPSO on three iris datasets are shown in Figures 16,17,18. From Figures 16,17,18, the fitness values in each fivefold optimization can be converged around a certain value in which the global best fitness and its corresponding particle is selected as the optimal solution. The details of the fitness and optimized Gabor parameters can be found in Table 3. Compared with the above-described Gabor filters, both Gabor energy and phase features achieve greater DI values and show more discriminative and informative Gabor features created by using optimized Gabor filters. Meanwhile, the optimized Gabor filters are best suited to extract the two types of local Gabor features simultaneously as a result of the definition of fitness. Due to the predefined Gabor filters as an empirical selection for the CASIA-I dataset, the improvements caused by the optimized Gabor filters on the CASIA-I and CASIA-V4Interval dataset are not so obvious. However, a greater DI value can be found at the JLUBR-IRIS dataset, which explains the necessity of the Gabor filter optimization for various datasets and validates our PSO- and BPSO-based Gabor filter optimization.

After achieving local Gabor energy and phase features and by using optimized Gabor filters, we attempted to train the SVR models to combine both matching scores of the two features to make an iris classification. The regression mode of the library for support vector machines (LibSVM) [56] tool is employed to train an SVR model. We tried four kernels and checked a grid of $\beta_{i}^{*}, \beta_{i}$ parameters using the simple tool LibSVM provided. Table 4 shows the DI values of the fused scores from four types of SVR kernels on three datasets. According to Table 4, the DI values of the fused scores showed a great improvement compared with the DI valves produced by a single Gabor feature. None of the best DI values from the SVR models with the linear kernel among three datasets reflect the non-linear correlation of the Gabor energy and phase features. The DDHs of the fused scores based on the corresponding trained SVR model on three datasets are shown in Figures 19,20,21. From Figures 19,20,21, the fused values from the authentic/imposter comparisons are much closer to the authentic label (0)/imposter label (1). Therefore, we can select a reasonable threshold to make the final decision. We draw the receiver operating characteristic (ROC) curves of a single feature by non-optimal Gabor filters, a single feature by optimized Gabor filters, and a fusion as shown in Figures 22,23,24. As shown in Figures 22,23,24, the best performance in this paper on each dataset had all been created by the fusion of multiple Gabor features. The EER from the optimized Gabor multi-feature fusion reached $0.07 \%, 0.461 \%$, and $0.344 \%$ on CASIA-I, CASIA-v4-Interval, and JLUBRIRIS datasets, respectively. The fusion method achieved almost perfect results on the CASIA-I dataset due to the good quality of its samples. The JLUBR-IRIS dataset is the most challenging iris dataset but had superior fusion results when compared to the CASIA-V4-Interval dataset because the more samples that are involved in the training

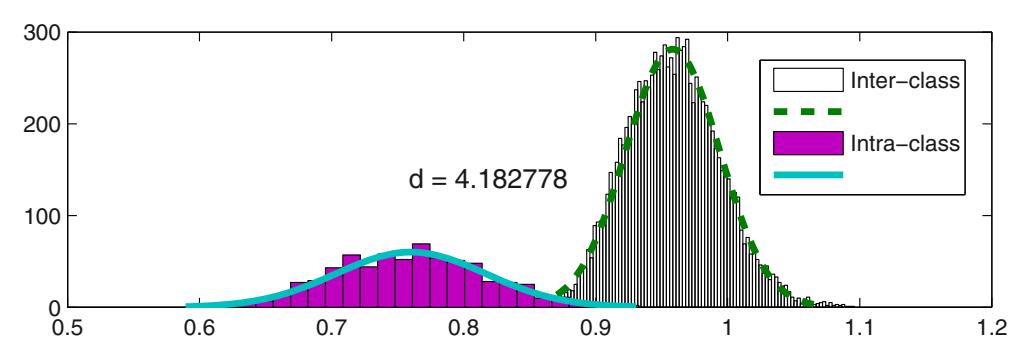

Figure 21 The DDH of fused scores on the JLUBR-IRIS dataset. 


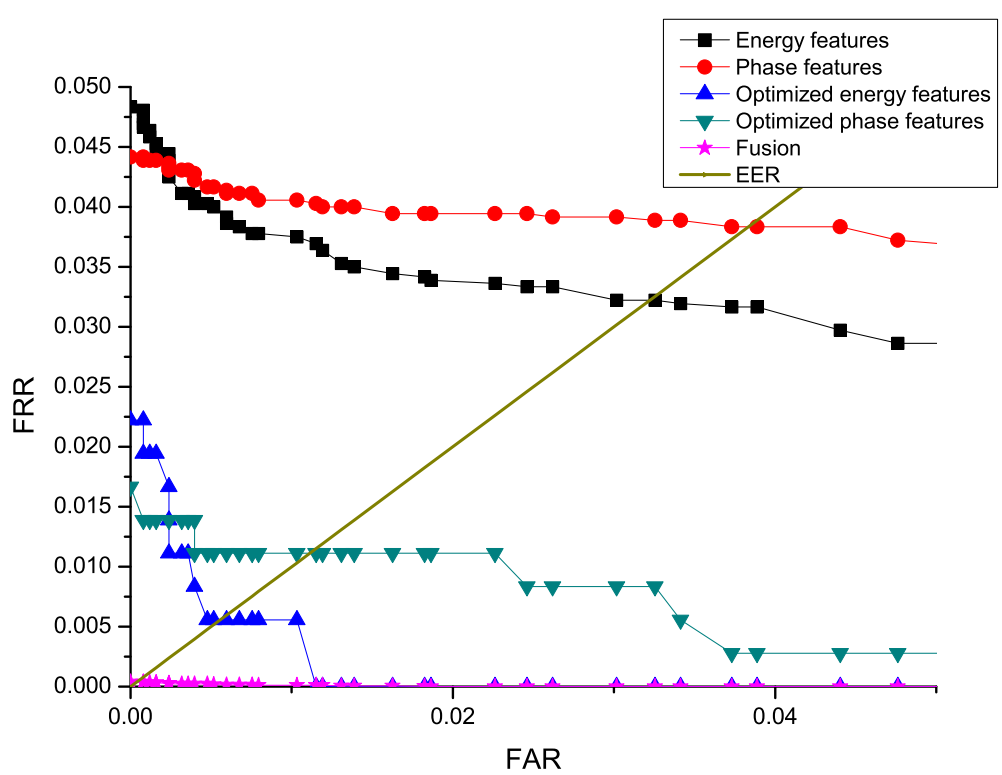

Figure 22 The ROC curves of different experiments on the CASIA-I dataset. The experiments include using a single feature by non-optimal Gabor filters, using a single feature by optimized Gabor filters, and using a fusion.

SVR model, the better mapping regressive function can be obtained. Thus, a better performance of the fused score can be further staged. In fusion experiments on the CASIA-V4-Interval dataset, we can only randomly extract five samples per class as the training set, and the rest is sampled as the test set due to its class size. Instead, due to the larger class size of the JLUBR-IRIS dataset, we could randomly extract 25 samples per class as the training set and the remaining 20 samples as the test set. Thus, through sufficient sample training, the more effective SVR model of JLUBR-IRIS dataset was established. Furthermore, from the overall ROC curves on each dataset, the local features can result in the lowest ROC curves by using optimized Gabor filters, which explains the necessity of Gabor filter optimization and multiple local Gabor feature fusion.

In order to show the sensitivity of our system to illumination variation, we performed six comparative

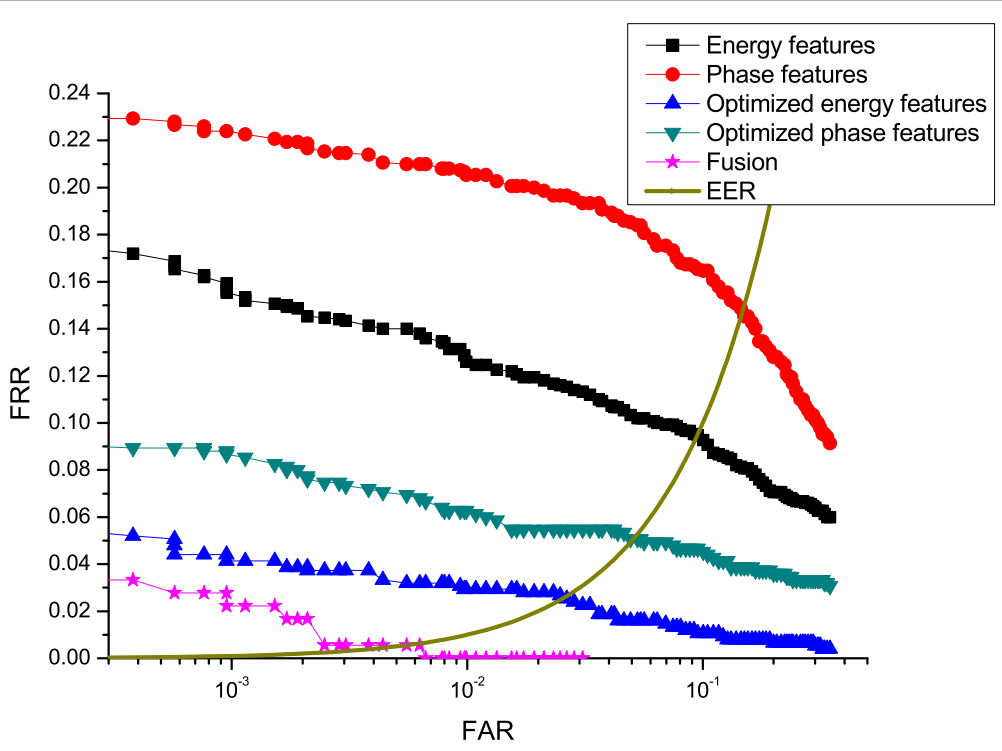

Figure 23 The ROC curves of different experiments on the CASIA-V4-Interval dataset. The experiments include using a single feature by non-optimal Gabor filters, using a single feature by optimized Gabor filters, and using a fusion. 


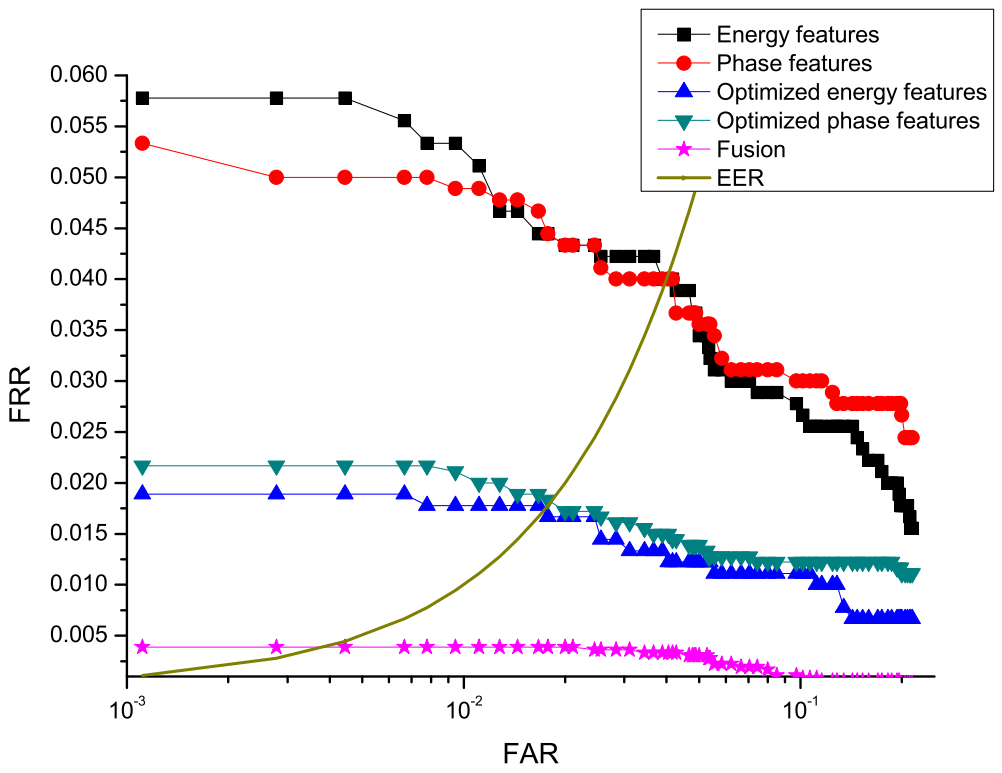

Figure 24 The ROC curves of different experiments on the JLUBR-IRIS dataset. The experiments include using a single feature by non-optimal Gabor filters, using a single feature by optimized Gabor filters, and using a fusion.

experiments with test images under various illumination levels on the JLUBR-IRIS dataset. Taking advantage of its different acquisition illuminations and adequate iris images (an example can be found in Figure 5), we form random samples from each pattern class under different illumination levels in an increasing way as the test set in the experiments, but the amount of the test set in each experiment still remains constant. These test samples are used to extract their multiple local Gabor features using optimal Gabor filters and to generate the fused scores via the above-described trained SVR model. Their ROC curves demonstrate the effect of variance of illumination levels on iris recognition rate in our

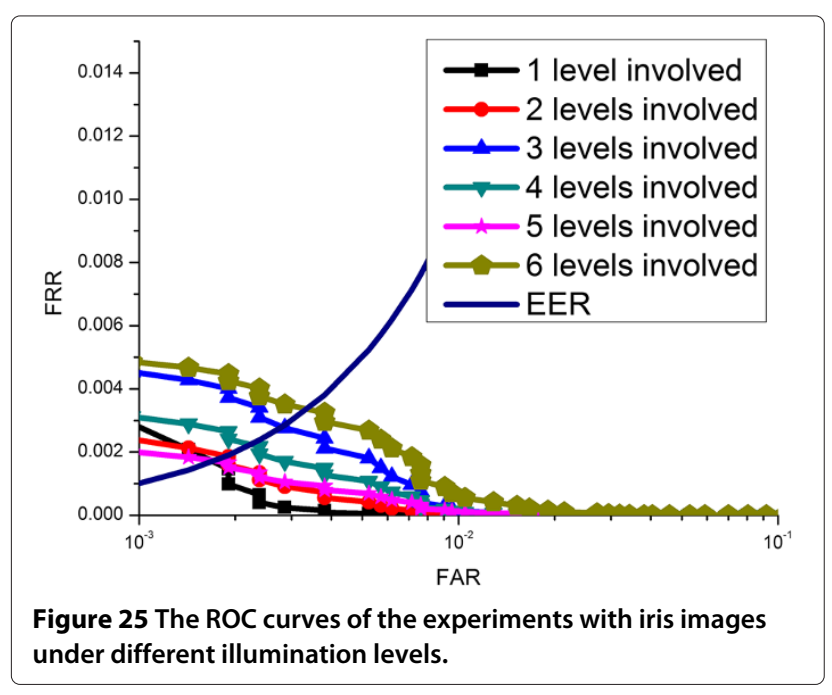

proposed system. It can be observed from Figure 25 that the performance of our multiple Gabor features and fusion scheme exhibits highly illumination invariance when illumination variation exists in the test samples. Due to the different iris samples among different experiments, they produce not identical but parallel ROC curves. The experiment with iris images under six different illuminations also can achieve comparable recognition performance to the ones with iris samples under fewer illumination levels. It illustrates that our system may be capable of performing illumination invariation in iris recognition by means of the adopted DC-free Gabor kernel, optimal Gabor filters, and fusion of multiple Gabor features.

According to the literature [54], Ma et al. realized the iris recognition of Daugman [1], Boles and Boashash [5], and his own proposed algorithm on the CASIA-I dataset. We combined our result on the CASIA-I dataset with the results of $\mathrm{Ma}$ et al. and compared them as shown in Table 5. From Table 5, we achieved an equal recognition

Table 5 A comparison of the different systems on the CASIA-I dataset

\begin{tabular}{lcc}
\hline Methodology & CRR (\%) & EER (\%) \\
\hline Daugman [1] & 100 & 0.08 \\
Boles et al. [5] & 92.64 & 8.13 \\
Ma et al. [56] & 100 & 0.07 \\
Wildes et al. [4] & N/A & 1.76 \\
Proposed & 100 & 0.07 \\
\hline
\end{tabular}




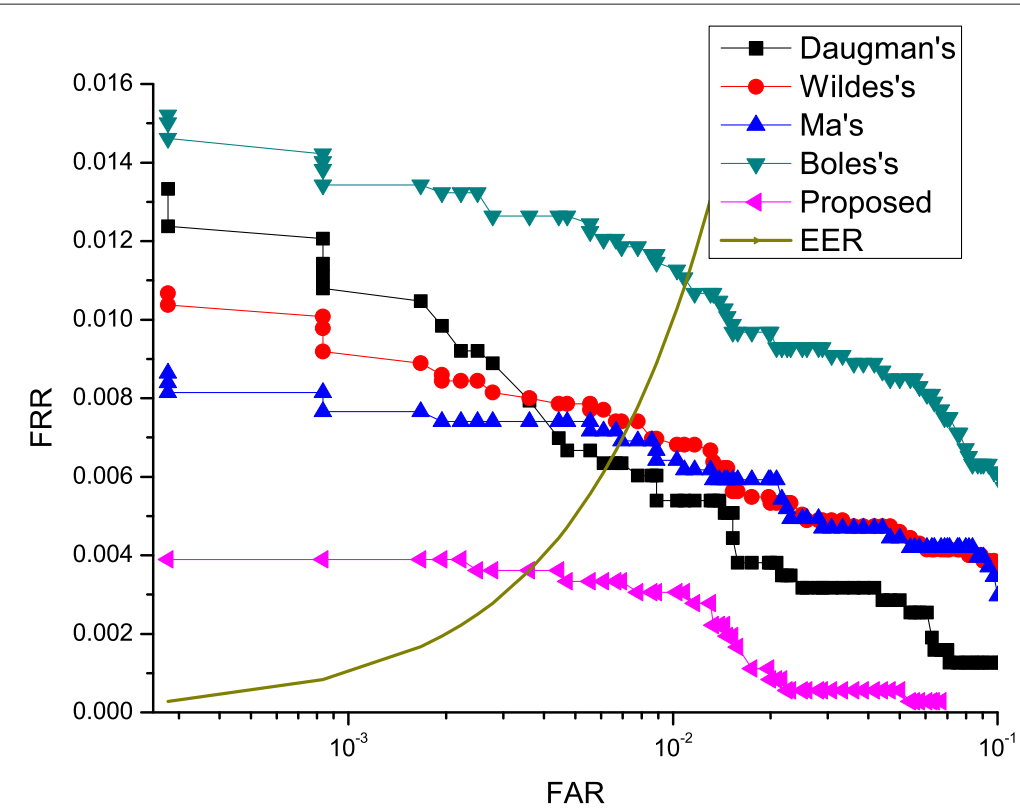

Figure 26 Performance comparisons of different systems on the JLUBR-IRIS dataset. The systems of Daugman, Wildes et al., Boles et al., Ma et al., and our system were compared.

rate compared to the system of Ma et al. on the CASIAI dataset. Compared to other approaches, our method involved richer texture representations to make a classification, which led to the best performance. Furthermore, a more challenging verification mode was adopted in our experiment as opposed to the identification mode adopted in [54]. Our results demonstrate a slight improvement compared to all other methods.

We further conducted comparative experiments on the JLUBR-IRIS dataset with the same experimental protocol. Figure 26 shows the ROC curves from these experiments. In Figure 26, our proposed method outperformed the other methods in terms of a lower ROC curve and EER value. Our method used optimal Gabor filters and multiple Gabor feature fusion to include more targeted and informative iris texture properties in the classification decision stage. The system of Boles et al. utilized little information along a concentric iris circle, while that of Wildes et al. made several low-pass filters to achieve

Table 6 Execution speeds of various operations for an iris match

\begin{tabular}{lc}
\hline Operation & Time $(\mathrm{msec})$ \\
\hline Localized and normalized iris region & 141 \\
Optimized Gabor filters filtering & 236 \\
Localized energy and phase feature generation & 5.2 \\
Multi-feature dissimilarity scores of two irises & 2.9 \\
Gabor multi-feature fusion decision on trained SVR & 3.2 \\
\hline
\end{tabular}

The speeds were taken using an Intel Core2 Quad Q9400 2.66-GHz processor. more distinctive features. Ma et al. encoded a local shape position using a particular class of wavelet transformation. These methods require a proper predefined set of wavelets to consolidate its robustness and accuracy, and the lack of performance by these wavelets may be caused by unofficial wavelets in our experiments. The Gabor phase code of Daugman is more robust and distinctive when compared with those of the other existing methods. The experimental results show that our system had excellent performance as compared with Daugman's system, which uses fewer Gabor features and an unknown Gabor filter selection.

The execution time of our proposed iris recognition system should be divided into two parts: training and recognition. The training part, including Gabor filter optimization and SVR modeling, is time consuming. Fortunately, the training part can be performed in the period of limited use (such as system initialization or at midnight each day) and is almost oblivious for the use of iris recognition. The average iris matching speed on our Intel Core2 Quad Q9400 2.66-GHz processor is shown in Table 6. An execution time of $0.38 \mathrm{~s} /$ comparison of the two irises can be satisfied for the real-time iris recognition system.

\section{Conclusion}

This paper has introduced an iris recognition system with multiple local Gabor feature extractions and fusion. This system uses two types of Gabor features generated by dividing the Gabor response magnitude and phase to represent an iris. This system then trains an SVR model to fuse them at score level for identification. In the process 
of Gabor filtering, adopted Gabor filters are optimized by a proposed PSO and BPSO optimization method. Our system has the advantages of adaptively tuning Gabor parameters and embedding richer informative texture into features and non-linear fusion strategies. The experimental results on our self-developed JLUBR-IRIS, public CASIA-I, and CASIA-V4-Interval iris datasets indicate that our localized method can avoid blocking effects to save more information when compared with other existing localized ways. The discriminative ability of the Gabor features demonstrates that the optimized Gabor filters may match the most informative frequency band iris texture than the predefined filters. Our score fusion by an SVR model is superior to other single-feature methods in terms of DI and ROC curves. In this paper, we also compared the proposed method with other algorithms and proved its validity and superiority.

\section{Competing interests}

The authors declare that they have no competing interests.

\section{Acknowledgements}

In this paper, portions of the research use the CASIA-Iris V4 and CASIA I datasets collected by the Chinese Academy of Sciences Institute of Automation (CASIA).

Received: 2 April 2014 Accepted: 30 May 2014

Published: 19 June 2014

\section{References}

1. J Daugman, High confidence visual recognition of persons by a test of statistical independence. IEEE Trans. Pattern Anal. Mach. Intell. 15(11), 1148-1161 (1993)

2. J Daugman, How iris recognition works. IEEE Trans. Circuits Syst. Video Technol. 14(1), 21-30 (2004)

3. RP Wildes, Iris recognition: an emerging biometric technology. Proc. IEEE. 85(9), 1348-1363 (1997)

4. RP Wildes, JC Asmuth, GL Green, SC Hsu, RJ Kolczynski, JR Matey, SE McBride, A machine-vision system for iris recognition. Mach. Vis. Appl. 9(1), 1-8 (1996)

5. WW Boles, B Boashash, A human identification technique using images of the iris and wavelet transform. IEEE Trans. Signal Processing. 46(4), 1185-1188 (1998)

6. L Ma, T Tan, Y Wang, D Zhang, Personal identification based on iris texture analysis. IEEE Trans. Pattern Anal. Mach. Intell. 25(12), 1519-1533 (2003)

7. H Proenca, LA Alexandre, Toward noncooperative iris recognition: a classification approach using multiple signatures. IEEE Trans. Pattern Anal. Mach. Intell. 29(4), 607-612 (2007)

8. A Ross, AK Jain, Multimodal biometrics: an overview, in Paper presented at the 12th European signal processing conference, (Vienna, Austria, Sept 2004), pp. 1221-1224

9. Y Si, J Mei, H Gao, Novel approaches to improve robustness, accuracy and rapidity of iris recognition systems. IEEE Trans. Ind. Informatics. 8(1), 110-117 (2012)

10. J Huang, X You, Y Yuan, F Yang, L Lin, Rotation invariant iris feature extraction using Gaussian Markov random fields with non-separable wavelet. Neurocomputing. 73(4), 883-894 (2010)

11. R Kaushik, B Prabir, Optimal features subset selection and classification for iris recognition. EURASIP J. Image Video Process. 2008, 1-20 (2008)

12. J-C Chang, M-Y Huang, J-C Lee, C-P Chang, T-M Tu, Iris recognition with an improved empirical mode decomposition method. Optical Eng. 48(4), 047007-047021 (2009)

13. H Mehrotra, B Majhi, P Gupta, Robust iris indexing scheme using geometric hashing of SIFT keypoints. J. Netw. Comput. Appl. 33(3), 300-313 (2010)

14. J Raja Sekar, S Arivazhagan, S Shobana Priyadharshini, S Shunmugapriya, Iris recognition using combined statistical and co-occurrence multi-resolutional features. Int. J. Pattern Recognit. Artif. Intell. 27(1), 1356001 (2013)

15. T Tan, X Zhang, Z Sun, $H$ Zhang, Noisy iris image matching by using multiple cues. Pattern Recognit. Lett. 33(8), 970-977 (2012)

16. Y Gong, D Zhang, P Shi, J Yan, Optimal wavelength band clustering for multispectral iris recognition. Appl. Optics. 51(19), 4275-4284 (2012)

17. H Proenca, G Santos, Fusing color and shape descriptors in the recognition of degraded iris images acquired at visible wavelengths. Comput. Vis. Image Understand. 116(2), 167-178 (2012)

18. D Gabor, Theory of communication. Part 1: The analysis of information. J. Inst. Electr. Eng. 3. 93(26), 429-441 (1946)

19. J Climent, RA Hexsel, Iris recognition using AdaBoost and Levenshtein distances. Int. J. Pattern Recognit. Artif. Intell. 26(02), 1266001 (2012)

20. YZ Du, C Belcher, Z Zhou, Scale invariant Gabor descriptor-based noncooperative iris recognition. EURASIP J. Adv. Signal Process. 2010(1), $936512(2010)$

21. CC Tsai, JS Taur, CW Tao, Iris recognition using Gabor filters and the fractal dimension. J. Inf. Sci. Eng. 25(2), 633-647 (2009)

22. $L Y u, D$ Zhang, $K$ Wang, The relative distance of key point based iris recognition. Pattern Recognit. 40(2), 423-430 (2007)

23. L Ma, Y Wang, T Tan, Iris recognition based on multichannel Gabor filtering, in Paper presented at the fifth Asian conference on computer vision, vol. 1 (Melbourne, Victoria, Australia, 2002), pp. 279-283

24. M Nabti, A Bouridane, An effective and fast iris recognition system based on a combined multi-scale feature extraction technique. Pattern Recognit. 41(3), 868-879 (2008)

25. X Li, M Xie, A novel algorithm of human iris recognition, in Paper presented at the IEEE international symposium on communications and information technology (ISCIT), vol. 2 (Beijing, China, 2005), pp. 1231-1234

26. J Thornton, M Savvides, BVK Kumar, An evaluation of iris pattern representations, in Proceedings of the First IEEE International Conference on Biometrics: Theory, Applications, and Systems (BTAS) (IEEE Seoul, Korea, 2007), pp. 1-6

27. Z Lin, B Lu, Iris recognition method based on the optimized Gabor filters, in Proceeding on the 3rd International Congress on Image and Signal Processing (CISP), vol. 4 (IEEE Yantai, China, 2010), pp. 1868-1872

28. CT Chou, SW Shih, DY Chen, Proceedings of the 2006 International Conference on Intelligent Information Hiding and Multimedia (IEEE Computer Society Pasadena, CA, USA, 2006), pp. 403-406

29. CC Tsai, CW Tao, JS Taur, Iris recognition using Gabor filters optimized by the particle swarm algorithm. J. Electron. Imaging 18(2), 023009-023019 (2009)

30. SE Grigorescu, N Petkov, P Kruizinga, Comparison of texture features based on Gabor filters. IEEE Trans. Image Process. 11(10), 1160-1167 (2002)

31. TXu, X Ming, $X$ Yang, Gabor filter optimization design for iris texture analysis. J. Bionics Eng. 1(1), $72-78$ (2004)

32. L Wiskott, J-M Fellous, N Kuiger, Malsburg von der C, Face recognition by elastic bunch graph matching. IEEE Trans. Pattern Anal. Mach. Intell. 19(7), 775-779 (1997)

33. J Daugman, Statistical richness of visual phase information: update on recognizing persons by iris patterns. Int. J. Comput. Vis. 45(1), 25-38 (2001)

34. FH Wang, JQ Han, Information fusion in personal biometric authentication based on the iris pattern. Meas. Sci. Technol. 20(4), 045501 (2009)

35. Y-P Huang, S-W Luo, E-Y Chen, An efficient iris recognition system. Proc. First Int. Conf. Mach. Learn. Cybernet. 1(1), 450-454 (2002)

36. J-K Kamarainen, Feature extraction using Gabor fillers, Ph.D. Dissertation, Lappeenranta University of Technology, Lappeenranta, 2003

37. L Wiskott, J Fellous, N Kruger, Malsburg von der C, Face recognition by elastic bunch graph matching. IEEE Trans. Pattern Anal. Mach. Intell. 19(7), 775-779 (1997)

38. V Kyrki, JK Kamarainen, H Kalviainen, Simple Gabor feature space for invariant object recognition. Pattern Recognit. Lett. 25(3), 311-318 (2004)

39. J Bigun, JMH du Buf, N-folded symmetries by complex moments in Gabor space and their application to unsupervised texture segmentation. IEEE Trans Pattern Anal. Mach. Intell. 16(1), 80-87 (1994)

40. YL Lee, HC Kim, HW Park, Blocking effect reduction of JPEG images by signal adaptive filtering. IEEE Trans. Image Process. 7(2), 229-234 (1998)

41. J Kennedy, R Eberhart, Particle swarm optimization. Proc. IEEE Int. Conf. Neural Netw. 4(2), 1942-1948 (1995)

42. A Moraglio, C Chio Di, R Poli, ed. by M Ebner, M O’Neill, A Ekárt, L Vanneschi, and Al Esparcia-Alcázar, Lecture notes in Computer Science: 
geometric particle swarm optimisation, in Genetic Programming (Springer Berlin, 2007), pp. 125-136

43. Y Shi, R Eberhart, A modified particle swarm optimizer, in IEEE World Congress on Computational Intelligence: IEEE International Conference on Evolutionary Computation Proceedings (IEEE Anchorage, AK, USA, 1998), pp. 69-73

44. F Afshinmanesh, A Marandi, A Rahimi-Kian, A novel binary particle swarm optimization method using artificial immune system, in The International Conference on Computer as a Tool (EUROCON), vol. 1 (IEEE Belgrade, Serbia, 2005), pp. 217-220

45. J Kennedy, RC Eberhart, A discrete binary version of the particle swarm algorithm, in IEEE International Conference on Systems, Man, and Cybernetics: Computational Cybernetics and Simulation, vol. 5 (IEEE Orlando, FL, USA, 1997), pp. 4104-4108

46. A Kumar, A Passi, Comparison and combination of iris matchers for reliable personal authentication. Pattern Recognit. 43(3), 1016-1026 (2010)

47. G Santos, E Hoyle, A fusion approach to unconstrained iris recognition. Pattern Recognit. Lett. 33(8), 984-990 (2012)

48. D Basak, S Pal, DC Patranabis, Support vector regression. Neural Inf. Process. Lett. Rev. 11(10), 203-224 (2007)

49. B Scholkopf, A Smola, Learning with Kernels. (The MIT Press, Cambridge, 2001), pp. 121-189

50. V Vapnik, The nature of statistical learning theory. (Springer, New York,2000)

51. JLUBR-IRIS Database. http://biis.jlu.edu.cn/irisdatabase/, Accessed $20 \mathrm{Mar}$ 2013

52. Specification of CASIA Iris Image Database (ver 1.0). (Center for Biometrics and Security Research, Chinese Academy of Sciences, 2005). http://www. cbsr.ia.ac.cn/english//risDatabase.asp. Accessed March 2007

53. WB Dong, ZN Sun, TN Tan, ris matching based on personalized weight map. IEEE Trans. Pattern Anal. Mach. Intell. 33(9), 1744-1757 (2011)

54. L Ma, T Tan, Y Wang, D Zhang, Efficient iris recognition by characterizing key local variations. IEEE Trans Image Process. 13(6), 739-750 (2004)

55. Z Sun, TTan, X Qiu, ed. by D Zhang, AK Jain, Graph matching iris image blocks with local binary pattern, in Advances in Biometrics, vol. 3832 (Springer Berlin, 2005), pp. 366-372

56. CC Chang, CJ, LIBSVM: a library for support vector machines. ACM Trans. Intell. Syst. Technol. (TIST). 2(3), 27 (2011)

doi:10.1186/1687-6180-2014-95

Cite this article as: He et al.: Multiple local feature representations and their fusion based on an SVR model for iris recognition using optimized Gabor filters. EURASIP Journal on Advances in Signal Processing 2014 2014:95. 